\title{
The biologic behavior of squamous cervical carcinoma after neoadjuvant therapy (NAT) according to immunohistochemical expression of E-cadherin and CD44v6
}

\author{
Mihaela Madalina Gavrilescu ${ }^{1,2}$, Raluca Balan ${ }^{*}, 1,3$, Viorel Scripcariu ${ }^{1,2}$, Dan Ferariu², \\ Ludmila Lozneanu $^{1,4}$, Diana Popovici ${ }^{1,3}$, Cornelia Amalinei $i^{1,5}$ \\ 1"Grigore T. Popa” University of Medicine and Pharmacy, lași, Romania, ${ }^{2}$ Regional Institute of \\ Oncology lasi, "3"Elena Doamna” Obstetrics and Gynecology Clinical Hospital Iași, Romania, "“'Sf. \\ Spiridon" Emergency Clinical Hospital, Iași, Romania, ${ }^{5}$ Institute of Forensic Medicine lași, Romania
}

\begin{abstract}
The efficiency of neoadjuvant therapy in cervical carcinoma has been well demonstrated, although the cellular mechanisms of different response to this treatment have not been thoroughly investigated. The present study consists of 24 patients with cervical cancer, including 12 patients in stages IB-IIIB with first-line surgical treatment and 12 patients in stages IIB-IIIB, with neoadjuvant therapy followed by radical hysterectomy. The aim of our study was to assess the correlations between the alterations in E-cadherin and CD44v6 immunoexpression in cervical carcinoma, as a tool of evaluation the response to neoadjuvant therapy and its prognostic significance. The intensity of CD44v6 immunoexpression was higher in more aggressive tumors and E-cadherin immunoexpression was approximately constant among the cases with neoadjuvant therapy. Our results demonstrate that the evaluation of CD44v6 immunoexpression in cervical carcinomas is useful for the assessment of tumor response to neoadjuvant therapy and of tumor aggressiveness. The high level of $\mathrm{E}$ cadherin immunoexpression in tumors with neoadjuvant therapy reflects its involvement in the prevention of HPV oncoproteins action, with benefits on the outcome. A larger group of patients and a panel of antibodies, including CD4 and COX2, could provide a better characterization of the tumor response to neoadjuvant therapy, with a positive prognosis impact.
\end{abstract}

Keywords: E-cadherin, CD44v6, cervical carcinoma, neoadjuvant therapy.

\section{Introduction}

Cervical cancer represents the third malignancy among all neoplasia, being on the second place, in the female population, after the breast cancer. Cervical cancer accounted for approximately 275,000 deaths worldwide in 2008 [1].

Received: July 2016; Accepted after review: September 2016; Published: September 2016.

${ }^{*}$ Corresponding author: Assoc. Prof. Raluca Balan, MD, PhD, "Elena Doamna" Obstetrics and Gynecology Clinical Hospital, 49 Elena Doamna Street, 700398 lași, Romania.

E-mail: raluca.balan@umfiasi.ro
Although radiotherapy associated with cisplatin chemotherapy became the gold standard in locally advanced cervical cancer therapy, about $50 \%$ of all these patients have recurrences or metastases in the first 2 years after treatment initiation [2]. The assessment of neoadjuvant therapy (NAT) effectiveness requires the discovery of new tumor biological markers which could contribute to the implementation of new therapeutic strategies.

For an optimal treatment, the possible effects on survival before the initiation of neoadjuvant therapy have to be identified. It is considered that CD44 is involved in cell adhesion, cell activation, regulation of tumor 
growth, and apoptosis. Recently, the expression of CD44 variant 6 (CD44v6) has been the subject of several studies that have demonstrated its usefulness in tumor progression and prognosis of patients with cervical cancer, its expression being correlated with a negative prognosis [3]. From the clinical point of view, a decrease of CD44v6 immunoexpression is correlated with a favorable response to neoadjuvant therapy. Strong correlations between CD44v6 expression and lympho-vascular space invasion and lymph node metastases have been reported in the literature $[4,5]$. CD44v6 is an independent prognostic factor in incipient stages of cervical cancer [6].

Loss of intercellular interactions in cervical cancer is an important event for promoting invasion and tumor progression, E-cadherin being one of the most studied proteins involved in intercellular adhesion. It has been proven that E-cadherin expression decreases progressively from normal to neoplastic epithelium. Thus, the expression of E-cadherin and a-catenin (E-cadherin associated cytoplasmic molecule) could provide additional information for understanding the mechanism of progression of premalignant lesions to invasive cervical cancer [7].

The aim of our study was to assess the correlations between the alterations in Ecadherin and CD44v6 immunoexpression in cervical carcinoma, as a tool of evaluation the response to neoadjuvant therapy and its prognostic significance.

\section{Material and Methods}

The tissue sections were carefully selected from 24 patients with cervical cancer (diagnosed by routine histopathology), including 12 patients in FIGO stages IB-IIIB who received first-line surgical treatment and 12 patients in FIGO stages IIB-IIIB, with neoadjuvant therapy followed by radical hysterectomy.

The collected tissues were fixed for 24 hours in buffered formalin and processed for paraffin embedding. Superfrost Plus adhesive slides were used and the serial sections of 4 $\mu \mathrm{m}$ were deparaffinized with xylen and rehydrated with absolute ethanol $\left(96^{\circ}, 70^{\circ}\right)$ and distilled water.

E-cadherin and CD44v6 Epitope Retrieval Solution pH 6 (code RE7113, Novocastra, UK) and Epitope Retrieval Solution $\mathrm{pH} 8$ (code RE7116, Novocastra, UK) were used for immunohistochemistry. After blocking the endogenous peroxidase and non-specific binding, the sections were incubated with the primary antibodies, anti-E-cadherin mouse monoclonal antibody (clone 36B5, code PA0387, Novocastra, UK), ready-to-use solution and anti-CD44v6 mouse monoclonal antibody (clone vFF-7, code NCL-CD44v6, Novocastra, UK), dilution range 1:100. The polymers detection kit, Novolink Polymer Detection System (code REF150-CE, Novocastra, UK), was used for the visualization of the immune reaction, following its characteristic working protocol.

The immunoexpression of E-cadherin and CD44v6 was semi quantitatively analyzed by two pathologists, working independently and using the same criteria: the pattern of expression and the number of immunopositive cells. For the pattern of expression, the sections were quantified under light microscopy $(\times 20 \mathrm{HPF})$ with a 4-point scoring system, as follows: 1 = absent; 2 = focal heterogeneous, 3 = reduced homogeneous; 4 $=$ intense homogeneous. The number of immunopositive cells was assessed in five random fields $(\times 40 \mathrm{HPF})$, using also a 4 -point scoring system [8]:

1 = less than $25 \%$ immunopositive cells;

$2=26-50 \%$ immunopositive cells;

$3=51-75 \%$ immunopositive cells;

$4=>75 \%$ immunopositive cells.

The immunostaining was assessed in the cell membrane, but cytoplasmic localization was also recorded and scored (Table 1).

For the statistical data it was used SPSS 21.0 characteristic for medical research. Proper tests specific for different types of analyzed data were applied, as tests comparing average values of a parameter corresponding to several groups of data including ANOVA, Scheffé, Spjotvol/Stoline, and specific correlation tests for quantitative and qualitative variables, such as Pearson, square Chi $\left(X^{2}\right)$, Mantel - Haenszel, Fisher, Spearman, Kendall tau, and Gamma. After 
applying these tests, the main parameters of interest were discussed and conclusions were established according to their values. Thus, reference parameter $p$ calculated in the tests represents the level of significance, which was compared with the critical $p=0.05$, corresponding to a 95\% confidence, with significant values for $p<0.05 . x^{2}$ is a nonparametric test used for statistical determinations, where two or more samples randomly drawn from a population have a different frequency distribution between them.

Table 1. The functions and the localization of the antibodies

\begin{tabular}{lll}
\hline Biological marker & Functions & Location \\
\hline E-cadherin & Intercellular adhesion & membrane/cytoplasm \\
CD44v6 & Intercellular adhesion & membrane/cytoplasm \\
\hline
\end{tabular}

Results

\section{CD44v6 immunostaining}

CD44v6 expression showed membrane staining, sometimes cytoplasmic, with variable intensity of immune positivity. In all the examined cases and for all clinic and morphological parameters taking into consideration, there was no significant difference between the pattern of expression and the number of immunopositive cells; therefore the sum of the scores was performed
For both groups of tumors, which benefited or not of NAT, there was no significant correlation between CD44v6 expression and specific clinic and morphological parameters, such as tumor differentiation grade. However, it could be noticed that the immunostaining total score of CD44v6 (the sum of the immune expression and the number of positive cells scores) was higher in more aggressive tumors (Figure 1), exhibiting lympho-vascular invasion and lymph node involvement.

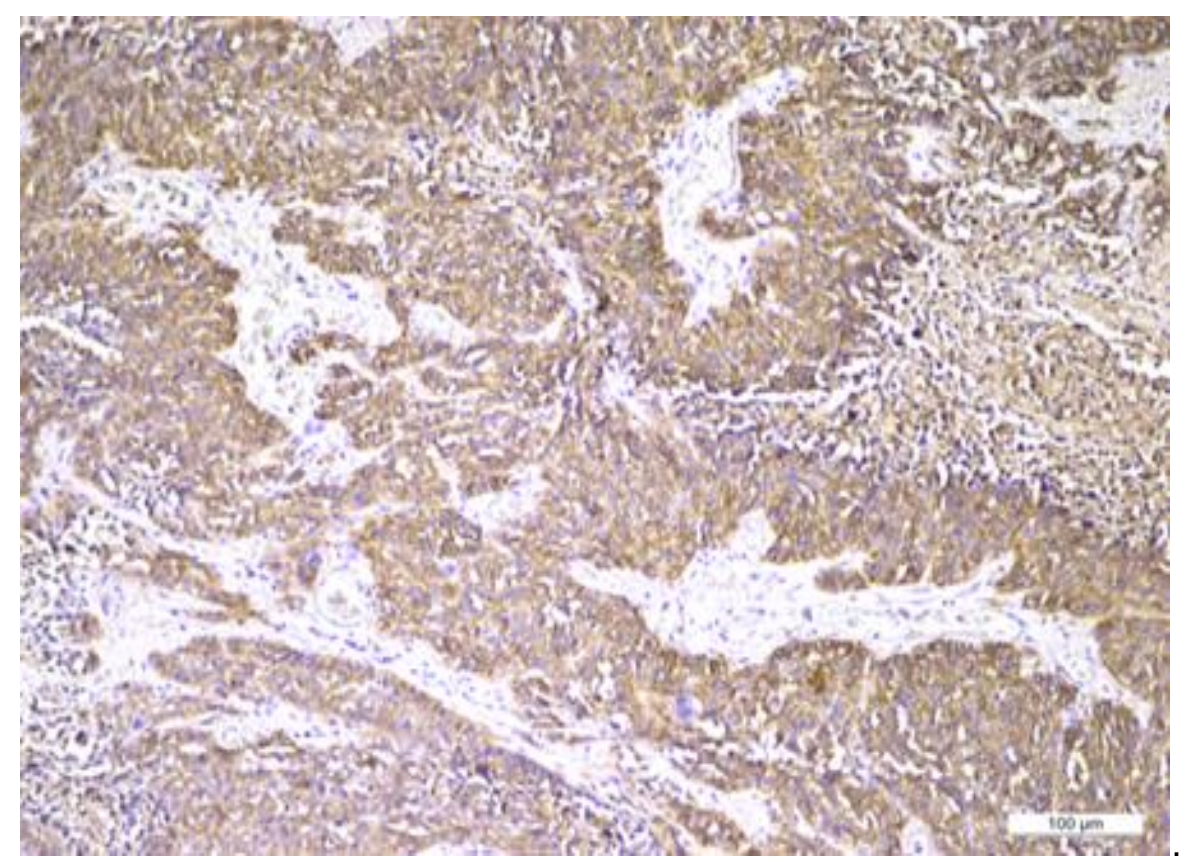

Fig. 1. SCC ulcerated infiltrative, with NAT: homogeneous immunopositivity in less than $51 \%$ tumor cells (IHC, Ab anti-CD44v6, x100) 
The tumors without NAT were numerically evenly distributed among those with immunostaining score 3 or 4 and the cases with score 1 or 2 . From the cases with score 4 , two cases had grade 2, but with lymphovascular invasion and lymph node involvement, one case was grade 2 , and one was grade 3 . Both cases with score 3 had grade 3 , one being also associated with endometrial carcinoma in situ.

Among the cases which presented score 2, 5 cases had grade 2, two presented lymph node involvement or lympho-vascular invasion, and one case was grade 3 (Table 2).

Table 2. CD44v6 and E-cadherin immunostaining sum of the scores in the studied cases

\begin{tabular}{|c|c|c|c|c|}
\hline Nr. & Histologic type & NAT & CD44v6 & E-cadherin \\
\hline 1 & Squamous cervical carcinoma (SCC) grade 2 & - & 2 & 3 \\
\hline 2 & Nonkeratinized squamous cervical carcinoma grade 3 & - & 3 & 3 \\
\hline \multirow[t]{2}{*}{3} & Keratinized squamous cervical carcinoma grade 3 & - & 2 & 3 \\
\hline & Nonkeratinized squamous cervical cervical carcinoma & & & \\
\hline 4 & grade 3 & - & 3 & 3 \\
\hline 5 & Nonkeratinized squamous cervical carcinoma grade 2 & - & 4 & 3 \\
\hline 6 & Keratinized squamous cervical carcinoma grade 3 & - & 4 & 3 \\
\hline 7 & Keratinized squamous cervical carcinoma grade 2 & - & 4 & 3 \\
\hline 8 & Keratinized squamous cervical carcinoma grade 2 & - & 2 & 3 \\
\hline 9 & Keratinized squamous cervical carcinoma grade 2 & - & 4 & 2 \\
\hline 10 & Nonkeratinized squamous cervical carcinoma grade 2 & - & 2 & 4 \\
\hline 11 & Keratinized squamous cervical carcinoma grade 2 & - & 1 & 3 \\
\hline \multirow[t]{2}{*}{12} & Nonkeratinized squamous cervical carcinoma grade 2 & - & 2 & 3 \\
\hline & Keratinized squamous cervical carcinoma ulcerated & & & \\
\hline 13 & infiltrative grade 2 & + & 3 & 3 \\
\hline 14 & Nonkeratinized squamous cervical carcinoma grade 3 & + & 1 & 3 \\
\hline 15 & Nonkeratinized squamous cervical carcinoma grade 2 & + & 2 & 3 \\
\hline 16 & Nonkeratinized squamous cervical carcinoma grade 2 & + & 2 & 2 \\
\hline 17 & Nonkeratinized squamous cervical carcinoma grade 2 & + & 1 & 3 \\
\hline 18 & Nonkeratinized squamous cervical carcinoma grade 3 & + & 1 & 3 \\
\hline 19 & Nonkeratinized squamous cervical carcinoma grade 2 & + & 2 & 3 \\
\hline 20 & Nonkeratinized squamous cervical carcinoma grade 2 & + & 1 & 3 \\
\hline 21 & Keratinized squamous cervical carcinoma grade 2 & + & 1 & 3 \\
\hline 22 & Nonkeratinized squamous cervical carcinoma grade 2 & + & 1 & 3 \\
\hline 23 & Keratinized squamous cervical carcinoma grade 3 & + & 1 & 3 \\
\hline 24 & Nonkeratinized squamous cervical carcinoma grade 2 & + & 3 & 3 \\
\hline
\end{tabular}


One case with score 1 had grade 2 and lympho-vascular invasion. Among the cases with NAT, 7 tumors presented a lower score, without a correlation with the pathological classification post NAT, the intensity of the immune reaction and the number of the immunopositive cells (Figure 2). Two cases with the highest score benefited of both radioand chemotherapy. Five out of 8 cases with score 1 had radio- and chemotherapy, and 3 cases had only radiotherapy, all of them presenting a partial response to NAT.

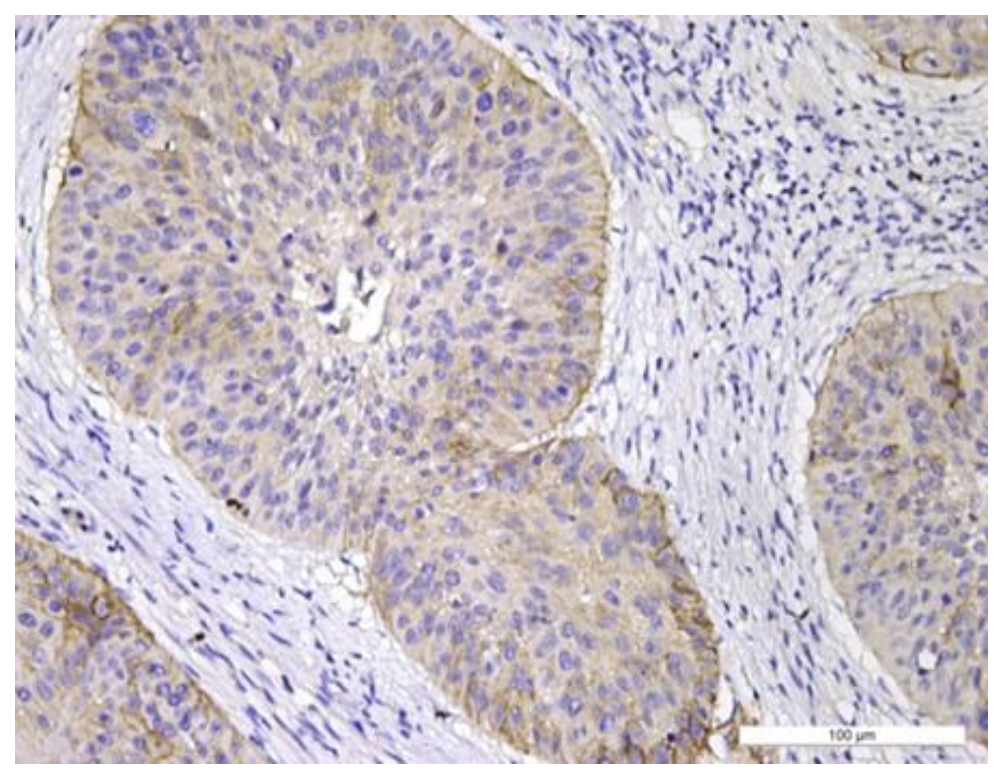

Fig. 2. SCC, with NAT: reduced homogeneous intense immunostaining in more than $75 \%$ tumor cells (IHC, $\mathrm{Ab}$ anti-CD44v6, x 200).

\section{Correlations between CD44v6 and parametrial involvement, lymph node (N) and lympho-vascular invasion}

There was a minimal relationship between CD44v6 and parametrial involvement $(\lambda$ $=0.500, p=0.064)$ (Table 3$)$, thus the patients with higher CD44v6 immunostaining score had higher parametrial invasion than the patients with lower CD44v6 immunostaining, which did not shad no expression of parametrial invasion (58.3\% vs. $8.3 \%)$.

In our study group, 8 patients had lymph node involvement. With the exception of patients with CD44v6 score 4, which showed a higher immunoexpression and a higher percent of immunopositive cells in patients without lymph node involvement $(18.8 \%$ vs. $12.5 \%)$, the scores 2 and 3 were more numerous in patients with lymph node involvement (37.5\% for both cases). However, the statistics emphasize that there is no significant relationship between CD44v6 and $\mathrm{N}$ $(\lambda=0.250, p=0.307)($ Table 3$)$.

Regarding the lympho-vascular invasion, the study results showed no significant association with CD44v6 immunostaining $(\lambda$ $=0.364, p=0.235$ ) (Table 3). CD44v6 score was higher in patients with lympho-vascular invasion $(45.5 \%, 27.3 \%$, and $18.2 \%$ for score 2,3 , and 4, respectively).

Table 3. Lambda test for the association of the CD44v6 values vs. $\mathrm{N}$ and lympho-vascular invasion, and parametrial involvement

\begin{tabular}{lll}
\hline & Lambda & $\mathbf{P}$ \\
\hline $\mathbf{N}$ & 0.250 & 0.307 \\
Parametrial involvement & 0.500 & 0.064 \\
Lympho-vascular invasion & 0.364 & 0.235 \\
\hline
\end{tabular}




\section{E-cadherin immunostaining}

E-cadherin immunostaining was positive in the tumor cells, as well as in the surface epithelium, but not in the connective tissue. In the surface epithelium, the immunostaining was mainly of membrane type, homogenous in the basal and parabasal layers, progressively decreasing toward the surface. As of CD44v6, in all examined cases and for all clinic and morphological parameters taking into consideration, there was no significant difference between the pattern of expression and the number of immunopositive cells of the E-cadherin, therefore the sum of the scores was performed.

Among the squamous carcinomas without NAT, there were 10 cases with score 3 , one case (grade 2 without lympho-vascular invasion) with score 2, and one case (grade 2 with lympho-vascular invasion) with the highest score (score 4). In some tumors of grade 2, the immunostaining was heterogeneous, with more than $50 \%$ immunopositive cells, with membrane staining in the central area of the tumor islands and more cytoplasmic staining toward the tumor periphery. However, the cases with grade 3 presented less than $51 \%$ immunopositive cells, with a lower intensity of the membrane staining and a higher cytoplasmic staining. Among the cases with NAT, the E-cadherin immunoexpression was approximately constant, 11 cases presenting score 3 and one case score 4 (Table 2).

\section{Correlations between E-cadherin, parametrial involvement, lymph node (N) and lympho-vascular invasion}

No significant relationship between Ecadherin immunostaining and parametrial involvement, lymph node $(\mathrm{N})$ and lymphovascular invasion $(p=0.307)$ was found in the study group (Table 4).

Score 2 of E-cadherin immunoexpression was more frequently noticed in the cases without lymph node involvement $(12.5 \%$ vs. $0 \%)$. Score 3 immunostaining was the same in both groups of patients (85.7\%). There was no patient from the study group without lymph node involvement with score 4.

E-cadherin immunostaining score was significantly higher (a score 3 for the pattern of expression in more than $51 \%$ immunopositive cells) in patients with parametrial involvement (91.7\% patients for score 3 ).

In an analogous manner, the patients with lympho-vascular invasion showed a higher Ecadherin immunostaining (90.9\% for score 3$)$.

Table 4. Lambda test for the association of the E-cadherin values vs. $\mathrm{N}$ and lympho-vascular invasion, and parametrial involvement

\begin{tabular}{lll}
\hline & Lambda & $\mathbf{P}$ \\
\hline $\mathbf{N}$ & 0.125 & 0.307 \\
Parametrial involvement & 0.083 & 0.307 \\
Lympho-vascular invasion & 0.091 & 0.307 \\
\hline
\end{tabular}

\section{Correlations between tumor grading, E- cadherin, and CD44v6}

There were statistically significant differences only for CD44v6 immunostaining, according with the degree of tumor differentiation $(p=0.048)$ (Table 5).

E-cadherin immunostaining was higher mainly for poorly differentiated tumors (G3) (100\% for score 3 ) (Figure 3 ). Score 4 was observed only in patients with moderately differentiated tumors (G2) $11.1 \%$ (Figure 4).
Well differentiated tumors presented more frequently score 2 of E-cadherin immunostaining $11.1 \%$. The statistical results showed no significant association between Ecadherin immunostaining and tumor grade (tau-c=0.068, $\mathrm{p}=0.350$ ) (Table 5).

CD44v6 analysis according to tumor differentiation grade reveals that the immunostaining score was higher mainly in patients with poorly or moderately differentiated tumors (tau-c $=0.313, p<0.05$ ) (Table 5). 


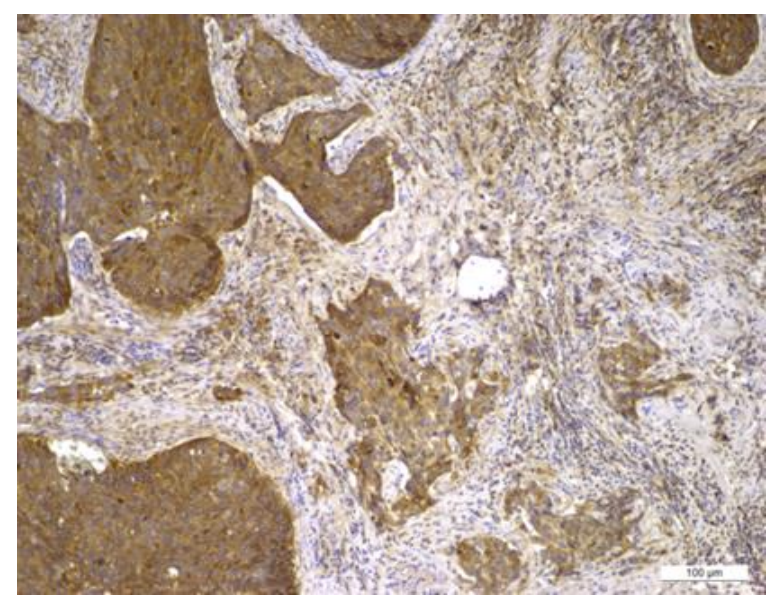

Fig. 3. Keratinized SCC, grade 3, without NAT: intense homogeneous immunostaining in more than $75 \%$ of tumor cells (IHC, Ab anti-E-cadherin, $\mathrm{x} 100)$.

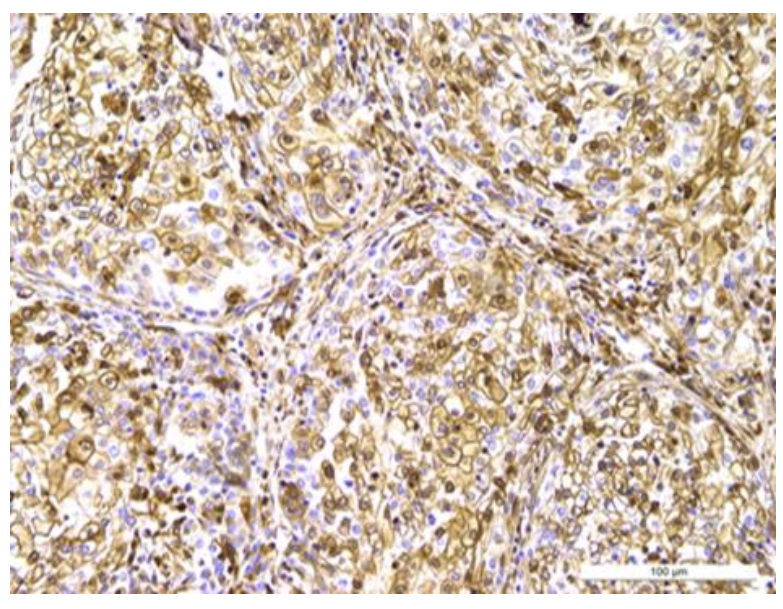

Fig. 4. Keratinized SCC, grade 2, without NAT: intense homogeneous immunostaining in more than $75 \%$ of tumor cells (IHC, Ab anti-E-cadherin, x200).

Table 5. Tau C test for tumor grading association values vs. E-cadherin and CD44v6

\begin{tabular}{lll}
\hline & Tau-c & P \\
\hline E-cadherin & 0.068 & 0.350 \\
CD44v6 & 0.313 & 0.048 \\
\hline
\end{tabular}

\section{Discussions}

The assessment of tumor prognosis has a significant importance for treatment planning, monitoring and patient counseling. Thus, it is unlikely that a single marker could represent the gold standard in predicting prognosis. In this regard, the assessment of a panel of antibodies in cervical carcinoma with different modes of action would be justified to improve prediction, with an impact upon the choice of optimal therapy.

It is considered that CD44 is involved in cell adhesion, cell activation and regulation of tumor growth. Several studies have shown the link between CD44 expression and apoptosis. From the clinical point of view, a decrease of CD44v6 immunoexpression is correlated with a favorable response to neoadjuvant therapy. There are many controversies about the role of CD44v6 as a prognostic factor. It is considered that aberrant expressions of CD44 isoforms are correlated with a poor prognosis in many cancers. In cervical carcinoma, CD44v6 isoform expression is correlated with a negative prognosis. It has been reported that cervical CD44v6 positive tumors respond better to chemotherapy compared with negative CD44v6 carcinomas [3]. These data are consistent with our results, in which the tumors that have benefited of NAT showed a significant lower CD44v6 immunoexpression, as compared with the patients without induction therapy.

There was no significant correlation between CD44v6 expression and certain clinico-morphological factors, such as parametrial involvement, lymph node and lympho-vascular invasion or even tumor differentiation grade, in our study.

CD44v6 immunostaining showed a significantly lower score in patients with induction therapy compared to patients who received neoadjuvant treatment $(p<0.05)$, results that are consistent with data reported by Costa et al. where the neoadjuvant treatment altered the expression of CD44v6 [3]. Although not statistically significant, patients with a higher score of CD44v6 immunoexpression had greater parameters invasion. Bouda et al. concluded that the patients with cervical cancer stage IB FIGO 
showed significant association between CD44v6 expression and histopathological type, depth of stromal invasion and lymphovascular invasion [9]. Although the results of this study revealed no significant differences between the expression of CD44v6 and the presence of positive nodes, tumor size or lympho-vascular invasion, we observed that the intensity of CD44v6 immunostaining and the percent of immunopositive cells were higher in more aggressive tumors, which had lympho-vascular invasion and lymph node involvement or tumors exceeding $4 \mathrm{~cm}$.

The survey data demonstrate CD44v6 expression involvement in assessment of response to neoadjuvant therapy and of aggressive character of cervical carcinomas. However, the lack of a significant correlation between CD44v6 immunoexpression and other clinico-morphological parameters suggests that this marker could be used within a panel of antibodies to characterize accurately the tumor response to neoadjuvant therapy.

Among the proteins involved in intercellular adhesion, E-cadherin is one of the most studied. It has been shown that Ecadherin expression gradually decreases from normal to neoplastic epithelium and invasive cervical carcinoma. Along with the evolution of cervical neoplasia, membrane E-cadherin expression is lost and cytoplasmic expression is gained. Increasing E-cadherin cytoplasmic immunoexpression was correlated with histological grade increase and lymph node metastases [10].

E-cadherin tumor cells and surface epithelium immunopositivity and lack of stromal expression in our study support other researchers' observations that increased cytoplasmic E-cadherin immunoexpression is correlated with tumor aggressiveness.

Epithelial cells can be converted to a mesenchymal phenotype through a multisequential reversible process called epithelial-mesenchymal transition (EMT). Cells undergoing this process lose their typical epithelial characteristics and acquire mesenchymal features. EMT is required for tumor differentiation, a step usually associated with an increased invasive potential and chemoresistance [11]. EMT induction and the associated spectrum of changes are triggered by the interference of numerous signaling pathways, with two common effects: (i) Ecadherin expression decrease (downregulation) and (ii) EMT associated genes expression. E-cadherin functions as tumor suppressor by mediating invasive suppressor abilities and maintaining the epithelial phenotype. E-cadherin expression is detected in well differentiated carcinomas, its expression being reduced in poorly differentiated tumors [12]. HPV E6 and E7 oncoproteins, involved in the cervical carcinoma pathogenesis, modulate the expression of EMT genes. Expression of E7 protein is associated with reduced expression of E-cadherin in dysplastic or malignant epithelial cells [11, 13]. Our findings of a higher E-cadherin expression in tumors associated with neoadjuvant therapy suggest its effectiveness in preventing the viral oncoproteins action, with positive impact on the outcome and NAT efficiency.

Regarding E-cadherin expression, the average staining intensity was lower in patients which benefited of neoadjuvant treatment compared with that noticed in patients who had surgical treatment. Statistical analysis results show that there is no significant association between E-cadherin expression and occurrence of positive nodes $(p=0.307)$. We were also unable to reach a statistically significant correlation between Ecadherin expression, parametrial involvement, the degree of tumor differentiation ( $p=0.350$ ), or lympho-vascular invasion, although it was noticed that E-cadherin immunostaining scoring 2 was found in $15.4 \%$ of patients which showed no lympho-vascular invasion and $0 \%$ in patients which had lympho-vascular invasion. Although this study is not statistically significant, we can emphasize that the average E-cadherin staining score was decreased in patients with tumors larger than $4 \mathrm{~cm}$. Ancuţa et al. found a significant association between E-cadherin expression and tumor size $(p=0.0029)$, relapse rate $(p=$ $0.002)$, and disease-free interval ( $p=0.003$ ). The authors demonstrated that $75 \%$ of patients with cervical cancer who had no Ecadherin expression and only $37.5 \%$ of the patients with positive reaction to $\mathrm{E}$-cadherin 
have relapses within 5 years from the time of diagnosis [14]. Cheng et al. demonstrates that decreased expression of E-cadherin and $\beta$ catenin were correlated with the degree of tumor differentiation, metastasis and recurrence in patients with cervical cancer, while vimentin overexpression was inversely correlated with the above mentioned parameters. The authors concluded that the association between E-cadherin decrease and vimentin increase can be a useful indicator for evaluation of the recurrence rate and survival [15].

Loss of E-cadherin membrane expression and increase of its diffuse cytoplasmic immunoexpression are correlated on one hand with increased tumor aggressiveness and on the other hand with a favorable response to neoadjuvant therapy.

The limitations of our study have been determined by the relatively reduced number of patients. In perspective, in order to validate and consolidate our data, an extension of the batch of patients with an assessment of a panel of tumor markers, including CD4 and

\section{References}

1. Ferlay J, Shin HR, Bray F, et al. Estimates of worldwide burden of cancer in 2008: GLOBOCAN 2008. Int J Cancer 2010; 127:2893-2917.

2. Nagy VM, Buiga R, Brie I, et al. Expression of VEGF, VEGFR, EGFR, COX-2 and MVD in cervical carcinoma, in relation with the response to radio-chemotherapy. Rom $J$ Morphol Embryol 2011; 52:53-59.

3. Costa S, Terzano P, Bovicelli A, et al. CD44 isoform $6(\mathrm{CD} 44 \mathrm{v} 6)$ is a prognostic indicator of the response to neoadjuvant chemotherapy in cervical carcinoma. Gynecol Oncol 2001; 80(1):67-73.

4. Biesold C, Kohler U, Horn LC, et al. CD 44 exon $v 6$ as a predictor of lymphatic metastases in cervical carcinoma--an immunocytochemical study of 94 cases. Arch Gynecol Obstet 1995; 256(3):147-153.

5. Kainz C, Tempfer C, Kohlberger $\mathrm{P}$, et al. Immunohistochemical detection of adhesion molecule CD44 splice variants in lymph node metastases of cervical cancer. Int $J$ Cancer 1996; 69(3):170-173.
COX2, could highlight possible correlations between their expression and clinic and morphological prognosis factors.

\section{Conclusions}

The evaluation of CD44v6 immunoexpression in cervical carcinomas is useful for the assessment of tumor response to neoadjuvant therapy and of tumor aggressive behavior. The high level of $\mathrm{E}$ cadherin immunoexpression in tumors associated with neoadjuvant therapy reflects its involvement in the prevention of HPV oncoproteins action, with benefits on the outcome and NAT efficiency. The increase of E-cadherin cytoplasmic immunoexpression is correlated with tumor aggressiveness and with a favorable response to neoadjuvant therapy. The increase of the batch of patients and the use of a panel of antibodies may provide an accurate characterization of the tumor response to neoadjuvant therapy and validate its prognosis value.

6. Speiser P, Wanner C, Tempfer C, et al. CD44 is an independent prognostic factor in earlystage cervical cancer. Int $J$ Cancer 1997; 74(2):185-188.

7. Carico E, Atlante M, Bucci B, et al. E-cadherin and alpha-catenin expression during tumor progression of cervical carcinoma. Gynecol Oncol 2001; 80:156-161.

8. Cruz MCFN, Pereira ALA, Lopes FF, et al. Immunohistochemical Expression of Ecadherin and CD44v6 in Squamous Cell Carcinomas of the Lower Lip and Tongue. Braz Dent J 2009; 20(1):64-69.

9. Bouda J, Boudova L, Hes O, et al. CD44v6 as a prognostic factor in cervical carcinoma FIGO stage IB. Anticancer Res 2005; 25:617-622.

10. Vessey CJ, Wilding J, Folarin N, et al. Altered expression and function of E-cadherin in cervical intraepithelial neoplasia and invasive squamous cell carcinoma. J Pathol 1995; 176(2):151-159.

11. Thiery JP, Sleeman JP. Complex networks orchestrate epithelial-mesenchymal transitions. Nat Rev Mol Cell Biol 2006; 7:131-142. 
12. Hirohashi $S$, Kanai $Y$. Cell adhesion system and human cancer morphogenesis. Cancer Sci 2003; 94:575-581.

13. Hellner $\mathrm{K}$, Mar J, Fang F, et al. HPV16 E7 oncogene expression in normal human epithelial cells causes molecular changes indicative of an epithelial to mesenchymal transition. Virology 2009; 391(1):57-63.
14. Ancuta E, Ancuta C, Cozma LG etal. Tumor biomarkers in cervical cancer: focus on Ki-67 proliferation factor and E-cadherin expression. Rom J Morphol Embryol 2009; 50:413-418.

15. Cheng $Y$, Zhou $Y$, Jiang $W$ et al. Significance of E-cadherin, beta-catenin, and vimentin expression as postoperative prognosis indicators in cervical squamous cell carcinoma. Hum Pathol 2012; 43:1213-1220. 